\title{
Separable operations on pure states
}

\author{
Vlad Gheorghiu* and Robert B. Griffiths \\ Department of Physics, Carnegie Mellon University, Pittsburgh, Pennsylvania 15213, USA
}

(Received 21 July 2008; published 29 August 2008)

\begin{abstract}
We show that the possible ensembles produced when a separable operation acts on a single pure bipartite entangled state are completely characterized by a majorization condition, a collection of inequalities for Schmidt coefficients, which is identical to that already known for the particular case of local operations and classical communication (LOCC). As a consequence, various known results for LOCC, including some involving monotonicity of entanglement, can be extended to the class of all separable operations.
\end{abstract}

DOI: 10.1103/PhysRevA.78.020304

PACS number(s): 03.67.Mn

\section{INTRODUCTION}

A separable operation $\Lambda$ on a bipartite quantum system is a transformation of the form

$$
\rho^{\prime}=\Lambda(\rho)=\sum_{k=1}^{N}\left(A_{k} \otimes B_{k}\right) \rho\left(A_{k} \otimes B_{k}\right)^{\dagger},
$$

where $\rho$ is an initial density operator on the Hilbert space $\mathcal{H}_{A} \otimes \mathcal{H}_{B}$. The Kraus operators $A_{k} \otimes B_{k}$ are arbitrary product operators satisfying the closure condition

$$
\sum_{k=1}^{N} A_{k}^{\dagger} A_{k} \otimes B_{k}^{\dagger} B_{k}=I_{A} \otimes I_{B},
$$

with $I_{A}$ and $I_{B}$ the identity operators. The extension to multipartite systems is obvious, but here we will consider only the bipartite case. To avoid technical issues the sums in (1) and (2) as well as the dimensions $D_{A}$ and $D_{B}$ of $\mathcal{H}_{A}$ and $\mathcal{H}_{B}$ are assumed to be finite.

Local operations with classical communication (LOCC) form a subset of separable operations in which the Kraus operators $A_{k} \otimes B_{k}$ are restricted by the requirement that they be generated in the following fashion. Alice carries out an operation $\left\{A_{i}^{(1)}\right\}, \sum_{i} A_{i}^{(1) \dagger} A_{i}^{(1)}=I_{A}$, in the usual way with the help of an ancilla, the measurement of which yields the value of $i$, which is then transmitted to Bob. He uses $i$ to choose an operation $\left\{B_{j}^{(2, i)}\right\}$, the result $j$ of which is transmitted back to Alice, whose next operation can depend on $j$ as well as $i$, and so forth. While it is (fairly) easy to see that the end result after an arbitrary number of rounds is of the form (1), it is difficult to characterize in simple mathematical or physical terms precisely what it is that distinguishes LOCC from more general separable operations. Examples show that separable operations can be more effective than LOCC in distinguishing certain sets of orthogonal states [1], even in a system as simple as two qubits [2], but apart from this little is known about the difference.

What we demonstrate in Sec. II of this paper is that the ensemble $\left\{p_{k},\left|\phi_{k}\right\rangle\right\}$ produced by a separable operation acting on a pure state $|\psi\rangle[$ see (5)], satisfies a majorization condition (7), which is already known to be a necessary and sufficient

\footnotetext{
*vgheorgh@andrew.cmu.edu
}

condition for producing the same ensemble from the same $|\psi\rangle$ by LOCC. Among the consequences discussed in Sec. III are the following. A separable operation acting on a pure state can be "simulated" by LOCC; a necessary condition for a deterministic transformation $|\psi\rangle \rightarrow|\phi\rangle$ given in [3] can be replaced by a necessary and sufficient majorization condition; and certain entanglement measures are nonincreasing under separable operations. Section IV summarizes our main result and indicates some open questions.

\section{ENSEMBLES PRODUCED BY SEPARABLE OPERATIONS ON PURE BIPARTITE STATES}

\section{A. Majorization conditions}

Let $\left\{A_{k} \otimes B_{k}\right\}_{k=1}^{N}$ be a separable operation on $\mathcal{H}_{A} \otimes \mathcal{H}_{B}$, specified by $N$ Kraus operators satisfying the closure condition (2). Let $|\psi\rangle$ be a normalized entangled state on $\mathcal{H}_{A}$ $\otimes \mathcal{H}_{B}$ with Schmidt form

$$
|\psi\rangle=\sum_{j=1}^{D} \sqrt{\lambda_{j}}\left|a_{j}\right\rangle\left|b_{j}\right\rangle,
$$

where $D=D_{B}$, and we assume without loss of generality that $D_{A} \geqslant D_{B}$. Here $\left\{\left|a_{j}\right\rangle\right\}$ and $\left\{\left|b_{j}\right\rangle\right\}$ are orthonormal bases chosen so that the Schmidt weights (coefficients) $\lambda_{j}$ are in increasing order, i.e.,

$$
0 \leqslant \lambda_{1} \leqslant \lambda_{2} \leqslant \cdots \leqslant \lambda_{D}
$$

The separable operation acting on $|\psi\rangle$ will produce an ensemble $\left\{p_{k},\left|\phi_{k}\right\rangle\right\}_{k=1}^{N}$, where

$$
\left(A_{k} \otimes B_{k}\right)|\psi\rangle=\sqrt{p_{k}}\left|\phi_{k}\right\rangle
$$

and

$$
p_{k}=\left\langle\psi\left|A_{k}^{\dagger} A_{k} \otimes B_{k}^{\dagger} B_{k}\right| \psi\right\rangle .
$$

In [4] it was shown that such an ensemble $\left\{p_{k},\left|\phi_{k}\right\rangle\right\}_{k=1}^{N}$ can be produced from $|\psi\rangle$ by a suitable LOCC if and only if the majorization inequalities

$$
\sum_{k=1}^{N} p_{k} E_{n}\left(\left|\phi_{k}\right\rangle\right) \leqslant E_{n}(|\psi\rangle)
$$

hold for $1 \leqslant n \leqslant D$, where 


$$
E_{n}(|\psi\rangle)=\chi_{n}\left(\operatorname{Tr}_{A}|\psi\rangle\langle\psi|\right)=\sum_{j=1}^{n} \lambda_{j},
$$

and similarly for the $\left|\phi_{k}\right\rangle$. Here $\operatorname{Tr}_{A}(|\psi\rangle\langle\psi|)$ is the reduced density operator of $|\psi\rangle\langle\psi|$ on Bob's side, and $\chi_{n}(\cdot)$ is defined to be the sum of the first $n$ smallest eigenvalues of its argument. Note that we are assuming that $D=D_{B} \leqslant D_{A}$, because if $D_{B}$ were greater than $D_{A}$ the extra zero eigenvalues in $\operatorname{Tr}_{A}|\psi\rangle\langle\psi|$ would cause confusion when using $\chi_{n}$.

Our main result is the following.

Theorem 1. The ensemble $\left\{p_{k},\left|\phi_{k}\right\rangle\right\}_{k=1}^{N}$ can be produced by a bipartite separable operation acting on the normalized state $|\psi\rangle$ if and only if the majorization condition defined by the collection of inequalities in (7) is satisfied.

Proof. To simplify the proof we assume that $D_{A}=D_{B}=D$. If $D_{A}$ is larger, one always modifies each $A_{k}$ by following it with a suitable local unitary which has the result that as long as the Kraus operators are acting on a fixed $|\psi\rangle$ the action on the $A$ side takes place in a subspace of $\mathcal{H}_{A}$ of dimension $D$. These local unitaries do not change the Schmidt weights of the $\left|\phi_{k}\right\rangle$ or alter the closure condition (2). For more details about this "decoupling," see [3].

When the majorization condition (7) holds, the result in [4] guarantees the existence of a LOCC (hence separable operation) which will produce the ensemble out of $|\psi\rangle$. The reverse inference, that the ensemble $\left\{p_{k},\left|\phi_{k}\right\rangle\right\}_{k=1}^{N}$ defined in (5) and (6) satisfies (7), follows from noting that

$$
p_{k} E_{n}\left(\left|\phi_{k}\right\rangle\right)=\chi_{n}\left[\operatorname{Tr}_{A}\left(A_{k} \otimes B_{k}|\psi\rangle\langle\psi| A_{k}^{\dagger} \otimes B_{k}^{\dagger}\right)\right],
$$

and applying Theorem 2 below with $R=I_{A} \otimes I_{B}$, corresponding to (2), so $\|R\|=1$.

\section{B. A majorization theorem}

Theorem 2. Let $\mathcal{H}_{A}$ and $\mathcal{H}_{B}$ have the same dimension $D$, let $|\psi\rangle$ be some pure state on $\mathcal{H}_{A} \otimes \mathcal{H}_{B}$, and let $\left\{A_{k} \otimes B_{k}\right\}_{k=1}^{N}$ be any collection of product operators on $\mathcal{H}_{A} \otimes \mathcal{H}_{B}$. Then for every $1 \leqslant n \leqslant D$

$$
\sum_{k=1}^{N} \chi_{n}\left[\operatorname{Tr}_{A}\left(A_{k} \otimes B_{k}|\psi\rangle\langle\psi| A_{k}^{\dagger} \otimes B_{k}^{\dagger}\right)\right] \leqslant\|R\| \chi_{n}\left(\operatorname{Tr}_{A}|\psi\rangle\langle\psi|\right),
$$

where $\|R\|=\sup _{\|\omega\|=1} \| R|\omega\rangle \|$ is the largest eigenvalue of the positive operator

$$
R=\sum_{k=1}^{N} A_{k}^{\dagger} A_{k} \otimes B_{k}^{\dagger} B_{k} .
$$

Proof. By map-state duality [3,5,6], using the Schmidt bases of $|\psi\rangle$, we transform the state $A_{k} \otimes B_{k}|\psi\rangle$ to a map $A_{k} \psi \bar{B}_{k}$, where

$$
\psi=\sum_{j=1}^{D} \sqrt{\lambda_{j}}\left|a_{j}\right\rangle\left\langle b_{j}\right|
$$

denotes an operator mapping $\mathcal{H}_{B}$ to $\mathcal{H}_{A}$, and $\bar{B}_{k}=B_{k}^{T}$ is the transpose of $B_{k}$. The matrix of $\psi$ using the Schmidt bases of $|\psi\rangle$ is diagonal, with the entries on the diagonal in increasing order. (See Sec. II of [3] for more details on map-state duality.) Upon writing the partial traces as

$$
\begin{gathered}
\operatorname{Tr}_{A}|\psi\rangle\langle\psi|=\psi \psi^{\dagger}, \\
\operatorname{Tr}_{A}\left(A_{k} \otimes B_{k}|\psi\rangle\langle\psi| A_{k}^{\dagger} \otimes B_{k}^{\dagger}\right)=A_{k} \psi \bar{B}_{k} \bar{B}_{k}^{\dagger} \psi^{\dagger} A_{k}^{\dagger},
\end{gathered}
$$

the inequalities (10) become

$$
\sum_{k=1}^{N} \chi_{n}\left(A_{k} \psi \bar{B}_{k} \bar{B}_{k}^{\dagger} \psi^{\dagger} A_{k}^{\dagger}\right) \leqslant\|R\| \chi_{n}\left(\psi \psi^{\dagger}\right) .
$$

For some $n$ between 1 and $D$, write the diagonal matrix $\psi$ as

$$
\psi=\psi_{n}+\widetilde{\psi}_{n},
$$

where $\psi_{n}$ is the same matrix but with $\lambda_{n+1}, \lambda_{n+2}, \ldots$ set equal to zero, while $\tilde{\psi}_{n}$ is obtained by setting $\lambda_{1}, \lambda_{2}, \ldots, \lambda_{n}$ equal to zero. Lemma 1 , below, tells us that for each $k$,

$$
\chi_{n}\left(A_{k} \psi \bar{B}_{k} \bar{B}_{k}^{\dagger} \psi^{\dagger} A_{k}^{\dagger}\right) \leqslant \operatorname{Tr}\left(A_{k} \psi_{n} \bar{B}_{k} \bar{B}_{k}^{\dagger} \psi_{n}^{\dagger} A_{k}^{\dagger}\right)
$$

By map-state duality,

$$
\operatorname{Tr}\left(A_{k} \psi_{n} \bar{B}_{k} \bar{B}_{k}^{\dagger} \psi_{n}^{\dagger} A_{k}^{\dagger}\right)=\left\langle\psi_{n}\left|A_{k}^{\dagger} A_{k} \otimes B_{k}^{\dagger} B_{k}\right| \psi_{n}\right\rangle,
$$

where $\left|\psi_{n}\right\rangle$, the counterpart of $\psi_{n}$, is given by (3) with $D$ replaced by $n$. Inserting (17) in (16) and summing over $k$ [see (11)], we obtain

$$
\begin{aligned}
\sum_{k=1}^{N} \chi_{n}\left(A_{k} \psi \bar{B}_{k} \bar{B}_{k}^{\dagger} \psi^{\dagger} A_{k}^{\dagger}\right) & \leqslant\left\langle\psi_{n}|R| \psi_{n}\right\rangle \leqslant\|R\|\left\langle\psi_{n} \mid \psi_{n}\right\rangle \\
& =\|R\| \chi_{n}\left(\psi^{\dagger} \psi\right) .
\end{aligned}
$$

This establishes (14), which is equivalent to (10).

Lemma 1 . Let $A, B$, and $\psi$ be $D \times D$ matrices, where $\psi$ is diagonal with nonnegative diagonal elements in increasing order, and for some $1 \leqslant n \leqslant D$ let $\psi_{n}$ be obtained from $\psi$ by setting all but the $n$ smallest diagonal elements equal to 0 , as in (15). Then

$$
\chi_{n}\left(A \psi B B^{\dagger} \psi^{\dagger} A^{\dagger}\right) \leqslant \operatorname{Tr}\left(A \psi_{n} B B^{\dagger} \psi_{n}^{\dagger} A^{\dagger}\right) .
$$

Proof. The inequality

$$
\chi_{n}\left(A \psi B B^{\dagger} \psi^{\dagger} A^{\dagger}\right) \leqslant \operatorname{Tr}\left(P_{n} A \psi B B^{\dagger} \psi^{\dagger} A^{\dagger} P_{n}\right),
$$

where $P_{n}$ is a projector (orthogonal projection operator) of rank at least $n$, follows from the fact that for any Hermitian operator $T$ the sum of its $n$ smallest eigenvalues is the minimum of $\operatorname{Tr}\left(P_{n} T P_{n}\right)$ over such $P_{n}$ (see p. 24 of [7]). Choose $P_{n}$ to be the projector onto the orthogonal complement of the range of $A \tilde{\psi}_{n}$, where $\tilde{\psi}_{n}=\psi-\psi_{n}$, as in (15). The rank of $A \tilde{\psi}_{n}$ is no larger than the rank of $\widetilde{\psi}_{n}$, which is smaller than or equal to $D-n$. Thus the dimension of the range of $A \tilde{\psi}_{n}$ cannot exceed $D-n$, so the rank of $P_{n}$ is at least $n$. By construction, $P_{n} A \tilde{\psi}_{n}=0$, so

$$
P_{n} A \psi=P_{n} A\left(\psi_{n}+\tilde{\psi}_{n}\right)=P_{n} A \psi_{n} .
$$

Thus, with this choice of $P_{n}$ the right side of (20) is 


$$
\operatorname{Tr}\left(P_{n} A \psi_{n} B B^{\dagger} \psi_{n}^{\dagger} A^{\dagger} P_{n}\right),
$$

and this implies (19), since $P_{n} \leqslant I$ and $A \psi_{n} B B^{\dagger} \psi_{n}^{\dagger} A^{\dagger}$ is positive.

\section{CONSEQUENCES}

The following are some consequences of Theorem 1 .

(i) An ensemble $\left\{p_{k},\left|\phi_{k}\right\rangle\right\}$ can be produced by a separable operation acting on a bipartite entangled state $|\psi\rangle$ if and only if it can be produced by some LOCC acting on the same state $|\psi\rangle$.

(ii) For a given bipartite $|\psi\rangle$ and separable operation $\left\{A_{k} \otimes B_{k}\right\}_{k=1}^{N}$, there is another operation of the form $\left\{\hat{A}_{l} \otimes U_{l}\right\}_{l=1}^{M}$, where the $U_{l}$ are unitary operators (and the closure condition is $\left.\sum_{l=1}^{M} \hat{A}_{l}^{\dagger} \hat{A}_{l}=I_{A}\right)$, which produces the same ensemble when applied to $|\psi\rangle$. Here $M$ could be different from $N$, as two Kraus operators might yield the same $\left|\phi_{k}\right\rangle$. For more details about the relation between the $\left\{A_{k}, B_{k}\right\}_{k=1}^{N}$ set and the $\left\{\hat{A}_{l} \otimes U_{l}\right\}_{k=1}^{M}$ set, see [8].

(iii) A deterministic transformation $|\psi\rangle \rightarrow|\phi\rangle$ by a separable operation is possible if and only if $E_{n}(|\phi\rangle) \leqslant E_{n}(|\psi\rangle)$ for every $n$ between 1 and $D$, with $E_{n}(\cdot)$ defined in (8). This is often written as $\lambda_{\psi}<\lambda_{\phi}$, where $\lambda_{\psi}$ and $\lambda_{\phi}$ are vectors of the corresponding Schmidt weights. (This extends Theorem 1 in [3].)

(iv) The maximum probability of success for the transformation $|\psi\rangle \rightarrow|\phi\rangle$ by a separable operation is given by

$$
p_{\max }^{\operatorname{sep}}(|\psi\rangle \rightarrow|\phi\rangle)=\min _{n \in[1, D]} \frac{E_{n}(|\psi\rangle)}{E_{n}(|\phi\rangle)},
$$

where $E_{n}(\cdot)$ was defined in (8).

(v) An entanglement measure $E$ defined on pure bipartite states is nonincreasing on average under separable operations, which is to say that

$$
E(|\psi\rangle) \geqslant \sum_{k=1}^{N} p_{k} E\left(\left|\phi_{k}\right\rangle\right)
$$

if and only if it is similarly nonincreasing under LOCC.

(vi) Let

$$
\hat{E}(\rho)=\inf \sum_{i} p_{i} E\left(\left|\psi_{i}\right\rangle\right)
$$

with the infimum over all ensembles $\left\{p_{i},\left|\psi_{i}\right\rangle\right\}$ yielding the density operator $\rho=\Sigma_{i} p_{i}\left|\psi_{i}\right\rangle\left\langle\psi_{i}\right|$, be the convex roof extension of a pure state entanglement measure $E$ that is monotone on pure states in the sense of (24). Then $\hat{E}$ is monotone on mixed states in the sense that

$$
\hat{E}(\rho) \geqslant \sum_{k=1}^{N} p_{k} \hat{E}\left(\sigma_{k}\right)
$$

for any ensemble $\left\{p_{k}, \sigma_{k}\right\}$ produced from $\rho$ by separable operations.
The result (i) is an immediate consequence of Theorem 1, as the same majorization condition applies for both separable and LOCC. Then (ii), (iii), and (iv) are immediate consequences of known results, in [8-10], respectively, for LOCC. The result (v) is an obvious consequence of (i), whereas (vi) follows from general arguments about convex roof extensions; see Sec. XV.C.2 of [11].

\section{CONCLUSION}

We have shown that possible ensembles of states produced by applying a separable operation to a bipartite entangled pure state can be exactly characterized through a majorization condition, the collection of inequalities (7) for different $n$. These have long been known to be necessary and sufficient conditions for producing such an ensemble using LOCC, so their extension to the full class of separable operations is not altogether surprising, even if our proof is not altogether straightforward.

Connecting the full set of separable operations with the more specialized LOCC class immediately yields several significant consequences for the former, as indicated in the list in Sec. III, because much is already known about the latter. Of particular significance is that various entanglement measures are monotone, meaning they cannot increase, under separable operations-something expected on intuitive grounds, but now rigorously proved. Since such monotonicity under LOCC has long been considered a necessary, or at least a very desirable, condition for any "reasonable" entanglement measure on mixed states (see Sec. XV.B of [11]), one wonders whether monotonicity under separable operations, in principle a stronger condition, might be an equally good or even superior desideratum.

Our results apply only to bipartite states, but separable operations and the LOCC subclass can both be defined for multipartite systems. Might it be that in the multipartite case the ensemble produced by applying a separable operation to a pure entangled state could also be produced by some LOCC applied to the same state? It might be, but proving it would require very different methods than used here. There are no simple multipartite analogs of the Schmidt representation (3), the majorization condition (7), or map-state duality.

Even in the bipartite case we still know very little about separable operations which are not LOCC, aside from the fact that they exist and can be used to distinguish certain collections of orthogonal states more effectively than LOCC. The results in this paper contribute only indirectly to a better understanding of this matter: looking at what a separable operation does when applied to a single entangled state will not help; one must ask what it does to several different states.

\section{ACKNOWLEDGMENTS}

We thank $\mathrm{Li} \mathrm{Yu}$ for useful comments. The research described here received support from the National Science Foundation through Grant No. PHY-0456951. 
[1] C. H. Bennett, D. P. DiVincenzo, C. A. Fuchs, T. Mor, E. Rains, P. W. Shor, J. A. Smolin, and W. K. Wootters, Phys. Rev. A 59, 1070 (1999).

[2] R. Duan, Y. Feng, and M. Ying, e-print arXiv:0705.0795.

[3] V. Gheorghiu and R. B. Griffiths, Phys. Rev. A 76, 032310 (2007).

[4] D. Jonathan and M. B. Plenio, Phys. Rev. Lett. 83, 1455 (1999).

[5] K. Życzkowski and I. Bengtsson, Open Syst. Inf. Dyn. 11, 3
(2004).

[6] R. B. Griffiths, S. Wu, L. Yu, and S. M. Cohen, Phys. Rev. A 73, 052309 (2006).

[7] R. Bhatia, Matrix Analysis (Springer-Verlag, New York, 1997).

[8] H.-K. Lo and S. Popescu, Phys. Rev. A 63, 022301 (2001).

[9] M. A. Nielsen, Phys. Rev. Lett. 83, 436 (1999).

[10] G. Vidal, Phys. Rev. Lett. 83, 1046 (1999).

[11] R. Horodecki, P. Horodecki, M. Horodecki, and K. Horodecki, e-print arXiv:quant-ph/0702225. 